\title{
Pulsar kicks with modified Urca and electrons in Landau levels
}

\author{
Ernest M. Henley \\ Department of Physics, University of Washington, Seattle, Washington 98195, USA \\ Mikkel B. Johnson \\ Los Alamos National Laboratory, Los Alamos, New Mexico 87545, USA \\ Leonard S. Kisslinger \\ Department of Physics, Carnegie Mellon University, Pittsburgh, Pennsylvania 15213, USA
}

(Received 12 June 2007; published 6 December 2007)

\begin{abstract}
We derive the energy asymmetry given the protoneutron star during the time when the neutrino sphere is near the surface of the protoneutron star, using the modified Urca process. The electrons produced with the antineutrinos are in Landau levels due to the strong magnetic field, and this leads to asymmetry in the neutrino momentum, and a pulsar kick. The magnetic field must be strong enough for a large fraction of the electrons to be in the lowest Landau level; however, there is no direct dependence of our pulsar velocity on the strength of the magnetic field. Our main prediction is that the large pulsar kicks start at about $10 \mathrm{~s}$ and last for about $10 \mathrm{~s}$, with the corresponding neutrinos correlated with the direction of the magnetic field. We predict a pulsar velocity of $1.03 \times 10^{-4}\left(T / 10^{10} \mathrm{~K}\right)^{7} \mathrm{~km} / \mathrm{s}$, which reaches $1000 \mathrm{~km} / \mathrm{s}$ if $T \simeq 10^{11} \mathrm{~K}$.
\end{abstract}

DOI: 10.1103/PhysRevD.76.125007

PACS numbers: 97.60.Bw, 97.60.Gb, 97.60.Jd

\section{INTRODUCTION}

The creation of neutron stars, often called pulsars, through neutrino cooling starting less than a second after the collapse of a massive star has long been of interest, with a number of processes contributing to neutrino production $[1,2]$. The strong interaction treatment of these processes was refined by Friman and Maxwell using perturbative one pion exchange and short-range interactions [3].

In recent years it has been observed that many pulsars move with much greater velocities than other stars in our galaxy. This is called the pulsar kick. See Ref. [4] for a review. Pulsars with velocities of more than $1500 \mathrm{~km} / \mathrm{s}$ have been observed. There has been a great deal of theoretical effort in attempts to explain the pulsar kicks. For many years a number of investigations of asymmetries in the hydrodynamics of core collapse have been carried out, and in recent years there have been a number of reviews [5-8]. Although some simulations have found possible pulsar velocities of $1000 \mathrm{~km} / \mathrm{s}$ or more, there is no clear proof that one can get the observed large pulsar velocities by the initial core collapse. There have been several calculations of possible asymmetry in the neutrinos produced in strong magnetic fields using the Urca process [9] and other processes $[10,11]$ during the first few seconds when the neutrinosphere has a radius of about $40 \mathrm{~km}$. However, the opacities and short mean- free paths of neutrinos in the neutrino atmosphere reduce the emission, and these processes cannot account for the large pulsar kicks $[12,13]$. There have also been calculations of pulsar kicks resulting from oscillation to sterile neutrinos [14-17], which can escape from the neutrino sphere during this early period. The recent MiniBooNE experiment [18] with previous
LSND results are not consistent with a single sterile neutrino, but allow models with two or more sterile neutrinos.

It has long been recognized that during the later period, when the neutrinosphere radius has been reduced to about $10 \mathrm{~km}$, the radius of the protoneutron star, the modified Urca process dominates the cooling of the protoneutron star [1,3]. It is also known that protoneutron stars have very large magnetic fields. In the presence of such fields, the electrons produced in the modified Urca process will be in Landau levels $[19,20]$.

In the present work we derive the asymmetric neutrino emissivity during the period when the neutrinosphere is just within the protoneutron star surface, and show that due to the electrons being in Landau levels pulsar velocities consistent with observations are obtained. In the present work the contribution from polarization of the nucleons is not included. Our work shows that for about a $10 \mathrm{~s}$ period starting at about $10 \mathrm{~s}$, when the modified Urca process dominates and the radius of the neutrinosphere is a fraction of a $\mathrm{km}$ less than the neutron star radius, the main neutrino emission is asymmetric, and during this period the high velocity pulsar kicks are generated. One of us presented a preliminary version of this work at the CosPA 2006 Symposium [21]. Also, the process of temperature equilibrium for the electrons in a strong magnetic field is being completed [22].

Our paper is organized as follows. In Sec. II we give the basic quantities in terms of which we calculate the emissivity. In Sec. III we explain the important differences between our formulation with the electrons in the lowest Landau level giving asymmetric momentum emissivity and the previous ones with no kick. As explained in Sec. III B, we incorporate the Landau wave function in 
the lepton trace and give the results of the calculation of the traces, explaining that with the contributing electrons all moving in the direction of the magnetic field the integration over the direction of the neutrino momentum is not present. In Sec. III D we give our results for the asymmetric emissivity that gives the pulsar kick. In Sec. we evaluate the essential ingredients of protoneutron star structure that is needed for obtaining our final numerical result, given in Eq. (26) and Fig. 4. In Sec. IV we present our conclusions. In the Appendix we give details of the calculation of the matrix elements, the nucleon and lepton traces, and the angular integrals.

\section{MODIFIED URCA PROCESS IN A STRONG MAGNETIC FIELD: LANDAU LEVELS}

The modified Urca process,

$$
n+n \rightarrow n+p+e^{-}+\bar{\nu}^{e},
$$

for cooling of protoneutron stars has been treated in many publications [1-3]. In a detailed calculation [3], the one pion exchange (OPE) and a short-range interaction were used for the nuclear interaction, illustrated in Fig. 1.

Diagrams (1), (2), and (3) are for neutral and charged pion exchange, diagrams (4), (5), and (6) are the exchange diagrams, and (7) is the short-range diagram. It can be shown that for our calculation of the asymmetric neutrino emissivity the short-range $n-n$ interaction is negligible, so we only consider the OPE diagrams here.

\section{URCA DIAGRAMS}
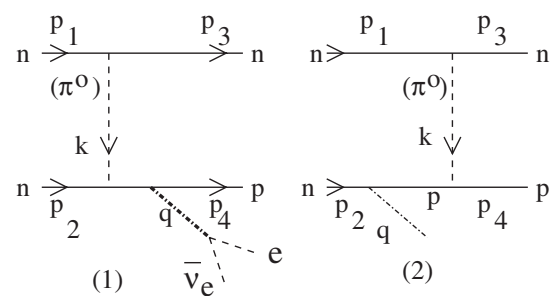

(2)

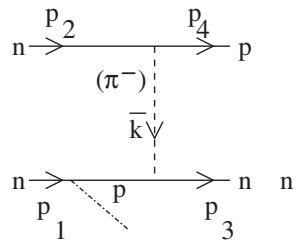

(3)
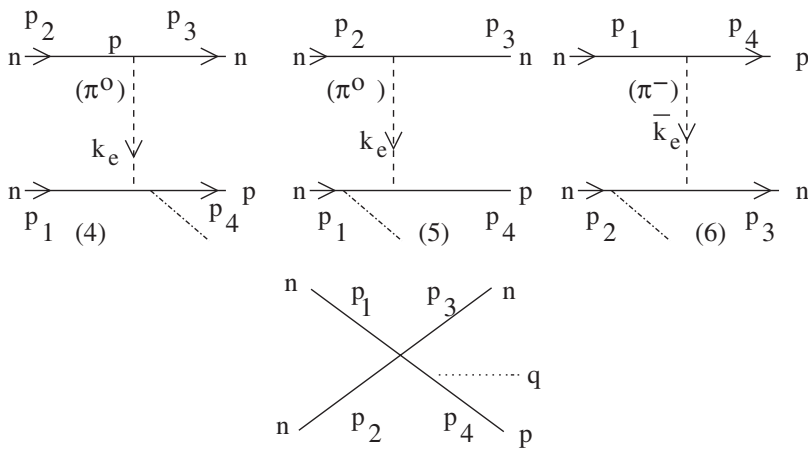

(7)

FIG. 1. Modified Urca diagrams with OPE and a short-range $n-n$ interaction.
The OPE factor is given by the standard nonrelativistic form, while the weak interaction used for the nucleons is the nonrelativistic form of the standard model. As we shall show, only the weak axial interaction, $W_{A}$, needs to be considered:

$$
\begin{aligned}
V_{\mathrm{OPE}} & =-\frac{f^{2}}{m_{\pi}^{2}} \tau^{1} \cdot \tau^{2} \vec{\sigma}_{1} \cdot \vec{k} \frac{1}{k^{2}+m_{\pi}^{2}} \vec{\sigma}_{2} \cdot \vec{k} \\
W_{A} & =-\frac{G}{\sqrt{2}} g_{A} \chi_{p}^{\dagger} \vec{l} \cdot \vec{\sigma} \chi_{n} \\
l_{\mu} & =\bar{\Psi}\left(q^{e}\right) \gamma_{\mu}\left(1-\gamma_{5}\right) \Psi\left(q^{\nu}\right),
\end{aligned}
$$

with $\sigma, \tau$ the Pauli spin and isospin operators (1) and (2) refer to the two nucleons at pion vertices, $G=\frac{10^{-5}}{m_{n}^{2}}, g_{A}=$ 1.26, the $\chi$ are the nucleon spinors, and the lepton wave functions are $\Psi\left(q^{e}\right), \Psi\left(q^{\nu}\right)$, where $q^{e}$ and $q^{\nu}$ are the electron and antineutrino momenta, respectively, with the notation $q^{e} \equiv \vec{q}^{e}, q^{\nu} \equiv \vec{q}^{\nu}$. Another point that should be mentioned is that the inverse modified Urca processes which we neglect in the present work,

$$
n+p+e^{-} \rightarrow n+n+\nu^{e} \quad n+e^{-} \rightarrow n+\pi^{-}+\nu^{e},
$$

will also have the electrons in Landau levels and thereby contribute to the overall pulsar kick, as we show below.

\section{Landau levels and product matrix elements}

The neutrino wave function is a Dirac spinor, while the electrons in a very strong magnetic field are in Landau levels, which are similar to that of a Dirac particle in a plane wave state moving in the $z$ or $-z$ direction, where $\hat{z}$ is the direction of the magnetic field, and the motion in the transverse direction is of the form of a tightly bound state. For a discussion of Landau wave functions, see Refs $[19,20]$. These states are labeled by a principal quantum number, $n$, and spin and momentum.

An essential aspect of the present work is that a sizable fraction of the electrons are in the lowest Landau level $(n=0)$. One can estimate the fraction of electrons in this $n=0$ level from the energy gap between the $n=0$ and $n=1$ levels, and $T$. The protoneutron stars that receive a large velocity from the mechanism that we are considering have a large magnetic field strength, about $10^{15}$ to $10^{16} \mathrm{G}$ at the star surface at a time of about $10 \mathrm{~s}$, when the neutrinosphere is near the surface of the protoneutron star. From expressions given in Ref [20], the energy gap between the $n=0$ and $n=1$ levels is about $6.0 \mathrm{MeV}$. Our work, shown below, concludes that a temperature $T$ with $k T$ about $8.59 \mathrm{MeV}$ will yield a pulsar velocity of about $1000 \mathrm{~km} / \mathrm{sec}$. From this we can use basic thermodynamics to estimate that the occupation probability of the $n=1$ state is about 0.5 , and that the overall probability of the electron being in the $n=0$ level is about $40 \%$.

A crucial point is that the electron in the $n=0$ level has its spin in the $-z$ direction, which we show below causes 
all of the emitted neutrinos to be correlated with the $z$ direction, while electrons in the higher $n$ levels have both helicities, and give no net pulsar kick. Since we assume that all electrons produced via the modified Urca process are in the $n=0$ Landau level, this introduces an error of a factor of approximately 2, but as we shall see this is a small correction due to the very strong dependence of the resulting pulsar velocity on $T$, and the uncertainty in the magnitude of $T$. We include this 0.4 factor in our final result.

The lowest Landau level, with $n=0$ has the form

$$
\begin{aligned}
\Psi\left(q^{\nu}\right) & =u^{s, \text { Dirac }}\left(q^{\nu}\right) \equiv u^{s}\left(q^{\nu}\right) \\
\sum_{s} u^{s}(q) \bar{u}^{s}(q) & =\not 1+m \\
\Psi\left(q^{e}\right) & =\psi^{\text {Landau }, n=0}\left(q_{\perp}^{e}, q_{z}^{e}, \phi\right) \\
& =i(\sqrt{\gamma})^{-1} e^{-\left(q_{\perp}^{e}\right)^{2} /(2 \gamma)} u^{-}\left(q^{e}\right),
\end{aligned}
$$

where $u^{-}$is a negative helicity Dirac spinor, $\not q \equiv q^{\mu} \gamma_{\mu}$, and $\gamma=\delta m_{e}^{2} ; \delta=B /\left(2 B_{c}\right) \simeq B / 8 \times 10^{13}$ Gauss $\simeq 12.5$ to 125 for $B=10^{15}$ to $10^{16}$ Gauss. Using $E+m \simeq E$, the wave functions have the conventional normalization $\int \bar{\Psi} \Psi=1$. The vector weak interaction is not included in our calculation, as its contribution to the neutrino asymmetric emissivity is much smaller than the axial vector. The pulsar velocity is independent of the magnitude of $B$ except for the $n=0$ occupation probability.

Although the $z$-component of the electron momentum can be in the $+z$ or $-z$ direction, we shall show that there is no neutrino emission for the electron moving in the $-z$ direction, due to the vanishing of the lepton trace.

For the calculation of the neutrino emissivity and the pulsar momentum, one needs the traces of the product matrix elements. The axial product matrix element, $\left|M_{A}\right|^{2}$, is obtained by taking the nucleon traces over the product of the leptonic traces times the square of the weakstrong product:

$$
\begin{aligned}
\left|M_{A}\right|^{2}= & \operatorname{Tr}(\text { nucleon }) \mid \operatorname{Tr}(\text { lepton })\left[l_{i}^{\dagger} l_{j}\right] \\
& \times\left.\left[W_{A}\left(V_{\mathrm{OPE}}+\text { exchange }\right)\right]_{i j}\right|^{2},
\end{aligned}
$$

with $V_{\mathrm{OPE}}$ given in Eq. (2) and the $(i, j)$ indices are the spatial components of the lepton currents, defined in Eq. (2). This is treated in detail in the following section and in the Appendix.

We introduce the following notation:

$$
\begin{aligned}
k & =p_{1}-p_{3}, \quad p=p_{2}-p_{1}, \quad k^{e}=k+p, \\
\chi_{i} \chi_{i}^{\dagger} & \equiv \Lambda_{i}=\left(1+\sigma \cdot P^{i}\right) / 2, \\
A & =\left(\frac{f}{m_{\pi}}\right)^{2} \frac{G}{\sqrt{2}} g_{A} \frac{1}{\omega} \frac{1}{\left(k^{2}+m_{\pi}^{2}\right)}, \\
R(k) & =\frac{k^{2}+m_{\pi}^{2}}{\left(k^{e}\right)^{2}+m_{\pi}^{2}} .
\end{aligned}
$$

The factor $R(k)$ is used in exchange matrix elements.

\section{NEUTRINO ASYMMETRIC MOMENTUM EMISSIVITY}

With a strong magnetic field the electrons are in Landau levels. That is, the motion of the electron transverse to the magnetic field is compressed, and the electron is essentially a one-dimensional plane wave Dirac particle moving in the direction of the magnetic field with energies given as Landau state energies. With a very strong magnetic field, such as near the surface of the protoneutron star when the modified Urca process dominates neutrino emission, the electron will fall into the lowest Landau level, with the wave function given in Eq. (3). The polarization of the nucleons is a very small effect [1]. The electrons being in Landau levels gives asymmetry to the neutrino emissivity. As we shall now discuss, the standard formulation for obtaining the energy emissivity from the modified Urca processes leads to the momentum of the emitted neutrinos correlated with the $B$-field direction, and thus directly to the pulsar velocity.

\section{A. Neutrino asymmetric emissivity}

The neutrino emissivity is given in general form in many papers, e.g., see Ref [3]:

$$
\begin{aligned}
e^{\nu}= & \Pi_{i=1}^{4} \int \frac{d^{3} p^{i}}{(2 \pi)^{3}} \frac{d^{3} q^{\nu}}{2 \omega^{\nu}(2 \pi)^{3}} \int \frac{d^{3} q^{e}}{(2 \pi)^{3}} \\
& \times(2 \pi)^{4} \sum_{s_{i}, s^{\nu}} \frac{1}{2 \omega_{L}^{e}} \omega^{\nu} \mathcal{F} M_{A}^{\dagger} M_{A} \\
& \times \delta\left(E_{\text {final }}-E_{\text {initial }}\right) \delta\left(\vec{p}_{\text {final }}-\vec{p}_{\text {initial }}\right) \\
\left|M_{A}\right|^{2}= & M_{A-A}^{D-D}+M_{A-A}^{E-E}+M_{A-A}^{D-E}+M_{A-A}^{E-D},
\end{aligned}
$$

where $\mathcal{F}$ is the product of the initial and final Fermi-Dirac functions corresponding to the temperature and density of the medium. We use the terminology that $M_{A}$ is the axial matrix element, which is given as the sum over the six Feynman-like diagrams shown in Fig. 1, but keeping only the axial weak interaction, shown in Eq. (2); $M_{A}^{D}$ stands for the direct diagrams 1, 2, and 3 in Fig. 1, $M_{A}^{E}$ for diagrams 4 , 5 , and $6, M_{A-A}^{D-D}$ is the product of $\left(M_{A}^{D}\right)^{\dagger}$ and $M_{A}^{D} \cdot M_{A-A}^{E-E}$ and $M_{A-A}^{D-E}, M_{A-A}^{E-D}$, are analogously defined. The sum runs over the nucleon spins, $s_{i}$, and the neutrino spin. The electron is in the lowest Landau level, with its wave function given in Eq. (3).

As in Refs. [1-3] the nucleons and the electrons are in thermal equilibrium. In this present work we assume that the proton quickly reaches thermal equilibrium, while the process of the electron state transforming to the $n=0$ Landau state does not interfere with the proton reaching its Fermi momentum. Therefore we can use the values for the magnitudes of the nucleon and lepton momenta derived in [1-3].

In the present paper we neglect the polarization of the nucleons, so the $\Lambda_{i}=I / 2$ [see Eq. (5)]. Thus the entire asymmetry of neutrino emission, which causes the pulsar 
kick in the present work, arises from the electrons being in the lowest Landau level and the modified Urca process.

\section{B. Neutrino asymmetric momentum emissivity}

As seen from Eq. (6), our calculation of the asymmetric neutrino emissivity starts with the standard theory, except that the electron is in the lowest Landau level, rather than in the usual Dirac state. This turns out to be the crucial point. With the electron in the lowest Landau level, the energy emissivity is also the projection of the momentum emissivity along the $\hat{B}$ axis. That is, in the following sections of the present paper we shall show that the emissivity given by Eq. (6) is of the form

$$
\begin{aligned}
e^{\nu}\left(q^{\nu}\right)^{z} & =\text { angular and energy integrals } \times|M|^{2}\left(q^{\nu}\right)^{z} \\
& =P^{A S}\left(q^{\nu}\right)^{z} c=-p(n s) c,
\end{aligned}
$$

where $\operatorname{Tr}($ lepton $) \operatorname{Tr}($ nucleon $)\left|M_{A}\right|^{2} \equiv|M|^{2}\left(q^{\nu}\right)^{z}, \quad c=$ speed of light, $P^{A S}\left(q^{\nu}\right)^{z}$ is the projection of the momentum emissivity (momentum/volume/time) along the $z=$ $B$ direction, and $p(n s)$ is the momentum/volume/time of the protoneutron star. It should be noted that the form equivalent to that of Eq. (7) has been used by a number of authors studying pulsar kicks, such as Refs. [16,17]. An essential ingredient in our framework is that electrons in the lowest Landau level moving in the direction of the magnetic field are emitted while those moving in the opposite direction are not, which produces the asymmetric momentum emissivity. This arises from the lepton traces [see Eqs. (4), (A12), and (A13)], It is a straightforward exercise in Dirac algebra to show that for an electron in the lowest Landau level $\left.\left(I-\gamma_{5}\right) u^{-}\left(q^{e}\right) \bar{u}^{-}\left(q^{e}\right)\right]$ vanishes for $\hat{q}^{e} \cdot \hat{B}=-1$, so that we can write

$$
\begin{aligned}
\int d^{2} q_{\perp}^{e} \operatorname{Tr}\left[l_{i}^{\dagger} l_{j}\right] \simeq & 8 \pi E^{e}\left[\left(q^{\nu}\right)^{j} \delta_{i 3}+\left(q^{\nu}\right)^{i} \delta_{j 3}-\delta_{i j}\left(q^{\nu}\right)^{3}\right] \\
& \times\left(\hat{q}^{e}=\hat{B}=\hat{z}\right),
\end{aligned}
$$

which is Eq. (A14), derived in the Appendix. Note $\left(q^{\nu}\right)^{i}$ is the $i$ th component of $q^{\nu}$, and $\left(q^{\nu}\right)^{3}=\left(q^{\nu}\right)^{z}$. This shows that when deriving the momentum asymmetry we can assume that the electrons in the lowest Landau levels are moving in the direction of the magnetic field. We shall take $\vec{q}^{e}$ in the $z(B)$ direction in the remainder of this paper.
An important result of our work is that the pulsar velocity does not depend directly on the strength of the magnetic field, as is indicated in Eq. (A14). This can be seen from the fact that the integral over the transverse components of the electron in the lowest Landau level, $\int d q_{\perp}^{e} q_{\perp}^{3} e^{-\left(q_{\perp}^{e}\right)^{2} / 2 \lambda}=$ $2 \lambda$, gives a factor of $\lambda$ which cancels the factor of $1 / \lambda$ from the square of the electron wave function [see Eq. (3)]. Therefore the only dependence of our pulsar velocity on the magnetic field strength is the requirement that it is large enough that a sizable fraction of the electrons are in the $n=0$ Landau level.

Therefore, with the electrons produced with the neutrinos in the modified Urca process undergoing a transition to the lowest Landau level in the very strong magnetic field near the protoneutron star, the standard theory for energy emissivity also gives the emitted momentum of the neutrinos, and therefore the recoil velocity of the resulting neutron star. However, there is a great deal to discuss, including the calculation of the various traces and the effective volume and time for the modified Urca process to give a pulsar kick.

We start with the calculation of the traces of $\left|M_{A}\right|^{2}$. As an example, let us calculate the direct axial matrix element. Writing the product of matrix elements needed for the emissivity, as shown in Eq. (6), and using the notation given in Eq. (5), with $M_{1}, M_{2}$, and $M_{3}$ the (1), (2), and (3) diagram in Fig. 1,

$$
\begin{aligned}
M 1+M 2= & A \chi_{3}^{\dagger} \sigma \cdot k \chi_{1} \chi_{4}^{\dagger}[\sigma \cdot k, \sigma \cdot l]_{+} \chi_{2} \\
= & 2 A l \cdot k \chi_{3}^{\dagger} \sigma \cdot k \chi_{1} \chi_{4}^{\dagger} \chi_{2} \\
M_{3}= & -2 A \chi_{3}^{\dagger} \sigma \cdot k \sigma \cdot l \chi_{1} \chi_{4}^{\dagger} \sigma \cdot k \chi_{2} \\
M_{A}^{D}= & M 1+M 2+M 3 \\
= & 2 A l \cdot k \chi_{3}^{\dagger} \sigma \cdot k \chi_{1} \chi_{4}^{\dagger} \chi_{2} \\
& -\chi_{3}^{\dagger} \sigma \cdot k \sigma \cdot l \chi_{1} \chi_{4}^{\dagger} \sigma \cdot k \chi_{2} .
\end{aligned}
$$

Further results and the calculation of the $M_{A-A}^{D-D}, M_{A-A}^{D-D}$, and $M_{A-A}^{D-E}$ traces of the product matrix elements are given in the Appendix. It is important to note that the Landau wave function is contained in the lepton current, $l_{i}$.

From the Appendix, Eqs. (A6), (A11), and (A14), and defining $M_{A A}^{D E} \equiv M_{A-A}^{D-D}+M_{A-A}^{E-D}$

$$
\begin{aligned}
\int d^{2} q_{\perp}^{e} M_{A-A}^{D-D}= & \int d^{2} q_{\perp}^{e} \operatorname{Tr}\left[l_{i}^{\dagger} l_{j}\right] A^{2}\left(k^{2} k_{i} k_{j}+k^{4} \delta_{i j}\right)=8 \pi A^{2} E_{e} k^{2}\left(-\left(q^{\nu}\right)^{3} k^{2}+2\left(q^{\nu}\right)^{i} k_{i} k_{z}\right) \\
M_{A-A}^{E-E}= & R(k)^{2} M_{A-A}^{D-D}\left(k \rightarrow k^{e}\right) \\
\int d^{2} q_{\perp}^{e} M_{A A}^{D E}= & -\int d^{2} q_{\perp}^{e} \operatorname{Tr}\left[l_{i}^{\dagger} l_{j}\right] A^{2} R(k)\left[-\frac{5}{2} k \cdot k^{e}\left(k_{i} k_{j}^{e}+k_{i}^{e} k_{j}\right)+2 k_{i}^{e} k_{j}^{e} k^{2}+2 k_{i} k_{j}\left(k^{e}\right)^{2}\right. \\
& \left.-\left(k \times k^{e}\right)_{i}\left(k \times k^{e}\right)_{j}+\left(k \cdot k^{e}\right)^{2} \delta_{i j}\right] \\
= & -8 \pi A^{2} R(k) E_{e}\left[-5 k \cdot k^{e}\left(k_{z}^{e} k \cdot q^{\nu}+k_{z} k^{e} \cdot q^{\nu}\right)-2 q \cdot k \times k^{e}\left(k \times k^{e}\right)_{z}+4 k \cdot q^{\nu} k_{z}\left(k^{e}\right)^{2}+4 k^{e} \cdot q^{\nu} k_{z}^{e}(k)^{2}\right. \\
& \left.+3\left(q^{\nu}\right)^{z}\left(\left(k \cdot k^{e}\right)^{2}-k^{2}\left(k^{e}\right)^{2}\right)\right] .
\end{aligned}
$$


The next step in the calculation is to carry out the nucleon angular integrals.

\section{Angular integrals with $k$ and $k^{e}$ angles independent}

Following the prescriptions of Ref. [3] the magnitudes of the momenta are given by the Fermi momenta, so one only does integrals over the energies and angles of the momenta, and the momentum transfer of the pion in the direct term, $\vec{k}=\vec{p}_{1}-\vec{p}_{3}$ is introduced as an independent vector by inserting

$$
\int d^{3} \delta\left(\vec{k}-\vec{p}_{1}+\vec{p}_{3}\right)=1 .
$$

For the $D-D$ (direct-direct) term, the only angular integral is over the direction of $k$. For the $E-E$ and $D-E$ terms, however, one must deal with $\vec{k}^{e}=\vec{k}+\vec{p}$ [see Eq. (5)]. Using the $\delta$-functions, one can see that neither $\vec{k}^{e}$ nor $\vec{p}$ are completely independent of $\vec{k}$, but with the proton momentum being smaller than the neutron, following arguments in Ref. [3], it is a good approximation to assume that $\vec{k}^{e}$ and $\vec{k}$ are independent.

Using the results given in Eq. (9) for the traces of the products of the matrix elements, the integrals given in Eq. (A15), and the approximations $k=k^{e}$ and $R(k)=1$, with the notation $\int=$ angular integrals over $\vec{k}, \vec{k}^{e}$, and $q_{\perp}^{e}$ one finds

$$
\begin{aligned}
& \iint M_{A-A}^{D-D}=\iint M_{A-A}^{E-E}=-\frac{128}{3} \pi^{2} A^{2} k^{4}\left(q^{\nu}\right)^{z} q^{e} \\
& \iint M_{A A}^{D E}=\frac{32}{9} \pi^{2} A^{2} k^{4}\left(q^{\nu}\right)^{z} q^{e} .
\end{aligned}
$$

Recognizing that the nucleons are in thermal equilibrium, the magnitudes of the nucleon momenta are given by the Fermi momentum, $p_{F}$, and therefore the integrals over the magnitude of the four nucleon momenta are carried out via delta functions. Dropping the $E^{\nu}$ terms, this gives the final result for the traces and integrals over the axial product matrix element:

$$
\begin{aligned}
\iint\left|M_{A}\right|^{2}= & -0.81 \times 10^{3} A^{2} q^{e}\left(q^{\nu}\right)^{z} p_{F}^{4} \\
= & -0.81 \times 10^{3}\left(\frac{f}{m_{\pi}}\right)^{4} \frac{G^{2} g_{A}^{2}}{2 \omega^{2}} \\
& \times\left(\frac{p_{F}^{2}}{p_{F}^{2}+m_{\pi}^{2}}\right)^{2} q^{e}\left(q^{\nu}\right)^{z} .
\end{aligned}
$$

\section{Neutrino asymmetric emissivity}

From Eq. (6), based on the general ideas of Ref. [3], but with our formalism for the phase space integrals, with the angular integrations given by Eqs. (10) and (11) in Sec. III C, we obtain the neutrino asymmetric emissivity:

$$
\left(\epsilon^{A S}\right)^{\nu}=2 \iint\left|M_{A}\right|^{2} \frac{\left(m_{n}^{*}\right)^{3} m_{p}^{*}}{(2 \pi)^{9}} p_{F}(e) I .
$$

The energy integrals, $I$, are the same as those in Ref. [3]; and with $q^{e}=85 \mathrm{MeV}$, and $q^{\nu}=4.7 \mathrm{kT}$, we find

$$
I=9.04 \times 10^{2}(\mathrm{kT})^{8} .
$$

From Eqs. (11)-(13) we obtain

$$
\begin{aligned}
\epsilon^{A S} & \simeq 0.64 \times 10^{21}\left(\frac{T}{10^{9} \mathrm{~K}}\right)^{7} \mathrm{erg} \mathrm{cm}^{-3} \mathrm{~s}^{-1} \\
& =p_{n s} c\left(\text { volume }^{-1} \text { time }^{-1}\right),
\end{aligned}
$$

where $p_{n s}$ is the momentum given to the neutron star, the volume is the active region for the modified Urca process, and the time represents the time interval. $p_{n s}$ has the magnitude of $\epsilon^{A S} / c$ in the opposite direction of the net neutrino momentum.

Because of the short mean-free path of neutrinos within the neutrinosphere, the main asymmetric emission from the process we have proposed will take place in the volume between the neutrinosphere and the protoneutron star surface during the period when the neutrinosphere is just within the protoneutron star surface. At this time the temperature is expected to be in the range $10^{9} \mathrm{~K}<T<$ $10^{11} \mathrm{~K}$ for a period of $10 \mathrm{~s}$ starting at about $10 \mathrm{~s}$. Taking the neutrinosphere and protoneutron star to have radii $R^{\nu}$ and $R_{n s}$, respectively, in km units, we find for the momentum given to the pulsar for this period of $10-20 \mathrm{~s}$

$$
p_{n s} \simeq 0.43 \times 10^{27}\left(\frac{T}{10^{9} \mathrm{~K}}\right)^{7}\left(R_{n s}^{3}-\left(R^{\nu}\right)^{3}\right) \mathrm{gm} \mathrm{cm} \mathrm{s}^{-1},
$$

where we use the effective volume for neutrino emission as $V_{n s}-V_{\nu \text {-sphere }}=f \frac{4 \pi}{3}\left(R_{n s}^{3}-\left(R^{\nu}\right)^{3}\right)$, where $f$ is the fraction of neutrinos which escape without striking the neutrinosphere for various positions of emission. A rough guess is 0.5 , and by integrating $q_{z}^{\nu}$, where $z$ is defined in the direction of $B$, we find that $f=0.52$.

\section{Radius of neutrinosphere during modified Urca emission}

The final step in our derivation is to estimate the volume in which the neutrino emission takes place with the modified Urca process in a strong magnetic field. Referring to Eq. (23), we must find the radius of the neutrinosphere, $R^{\nu}$, assuming that the radius of the protoneutron star at this time is $10 \mathrm{~km}$.

To do this we use the spherical Eddington model, which has been used by a number of authors to study the neutrino atmosphere associated with the creation of a pulsar $[16,17,23,24]$. We follow the recent method of Barkovich et al. [17] to find the neutrinosphere radius, and estimate the time and temperature during which our process is taking place. See Ref. [25] for a review of the evolution of the birth of a neutron star. 
Our starting point is the energy-momentum tensor, $T^{\mu \nu}$ for the neutrinos with a distribution function $f^{\nu}(\vec{x}, \vec{k}, t)$ for each type of neutrino, giving an energy density $U$ and momentum density $\vec{F}$ :

$$
U=T^{00}=\int \frac{d^{3} k}{(2 \pi)^{3}} k_{0} f^{\nu} \quad F^{i}=T^{0 i}=\int \frac{d^{3} k}{(2 \pi)^{3}} k^{i} f^{\nu} .
$$

Making use of the Boltzmann equation, and recognizing that the neutrinos have a very short mean-free path, $\lambda^{\nu}$, the neutrino distribution can be written in terms of the equilibrium distribution $\left(f^{\mathrm{eq}}\right)^{\nu}(\vec{x}, \vec{k})$ as

$$
f^{\nu} \simeq\left(f^{\mathrm{eq}}\right)^{\nu}-\lambda^{\nu} \hat{k} \cdot \Delta\left(f^{\mathrm{eq}}\right)^{\nu} \quad\left(f^{\mathrm{eq}}\right)^{\nu}=\frac{1}{1+e^{\left(k-\mu^{\nu}\right) / T}},
$$

which form we have used to obtain the integrals in Eq. (16).

The mean-free path for each neutrino type in a medium of nucleons with density $\rho$ can be written as

$$
\lambda^{\nu}=\frac{1}{\chi^{\nu} k^{2} \rho},
$$

with the constant $\chi^{\nu}$ determined from the constants of the standard weak interaction model and the cross sections for each neutrino type. Assuming the spherical Eddington model, one finds for the energy and momentum densities, including the electron, muon, and tau neutrinos, for equilibrium temperature $T$ :

$$
U=\frac{7 \pi^{2}}{40} T^{4} \quad \vec{F}=-\frac{1}{36 \chi \rho} \frac{d T^{2}}{d r} \hat{r} .
$$

The time dependence of $T$ can be found from energymomentum conservation:

$$
\partial^{\nu} T^{0 \nu}=0 \quad \text { or } \quad \partial_{t} U+\Delta \cdot \vec{F}=0 .
$$

Using the equations of hydrostatic equilibrium, Barkovich et al. showed that the $T$ can be obtained from the first order differential equation,

$$
\frac{d \bar{T}}{d x}+b_{c} \frac{\left.\bar{T}^{2}-a(x)\right)}{\bar{T}^{2} x^{2}(1-a(x))}=0,
$$

with $\bar{T} \equiv T / T_{c}, x=r / R_{n s}$, and $a(x)$ depends on the constant $b_{c}$ and two other constants, as well as $\bar{T}$. We use the constants of Ref. [17], with $T_{c}=40 \mathrm{MeV}$, slightly altered for our expected luminosity in the 10-30 s interval when the modified Urca process dominates.

Our solutions are shown in Figs. 2 and 3.

In our solutions we find that $a(x) \ll 1, a(x) \ll \bar{T}$, $a(x) \simeq \bar{T}(x)^{2}(x \geq 1)$, and that the result for the mean-free path is

$$
\left(\lambda^{\nu}\right)^{-1} \simeq \bar{T}\left(\bar{T}^{2}-a\right) \mathrm{cm}^{-1} .
$$

With this solution the radius of the neutrinosphere can be obtained in terms of $R_{n s}$ from the relation

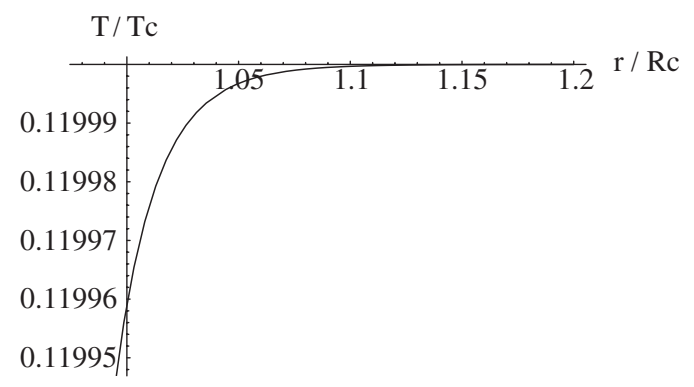

FIG. 2. Temperature vs radius near the star surface.

$$
\int_{R^{\nu} / R_{n s}}^{\infty} \bar{T}\left(\bar{T}^{2}-a\right) d x \simeq \frac{2}{3} \frac{\mathrm{cm}}{R_{n s}} .
$$

Using our solutions we find that for $R_{n s}=10 \mathrm{~km}$,

$$
R^{\nu} \simeq 9.96 \mathrm{~km},
$$

when the temperature is in the range $T \simeq 10^{10} \mathrm{~K}$, so that from Eq. (23) the neutron star momentum is

$$
p_{n s} \simeq 5.14 \times 10^{27} \mathrm{gm} \mathrm{cm} / \mathrm{s}\left(\frac{T}{10^{9}}\right)^{7}=M_{n s} v_{n s} .
$$

For a neutron star with the mass of the sun $=2 \times 10^{33} \mathrm{gm}$, including a factor of 0.4 for the $n=0$ occupation probability,

$$
v_{n s}=1.03 \times 10^{-4}\left(\frac{T}{10^{10}}\right)^{7} \mathrm{~km} \mathrm{~s}^{-1},
$$

giving a velocity of $v \simeq 1000 \mathrm{~km} / \mathrm{s}$ for $T \simeq 10^{11} \mathrm{~K}$, which is in the expected range. Figure 4 illustrates the velocity of the pulsar as a function of $T$.

Therefore we find that the modified Urca process can produce the observed velocities of $1000 \mathrm{~km} / \mathrm{s}$ or more during this period if the temperature is sufficiently high when the neutrinosphere is slightly within the protoneutron star due to the electrons being in Landau levels. These large pulsar kicks start about $10 \mathrm{~s}$ after the supernova collapse.

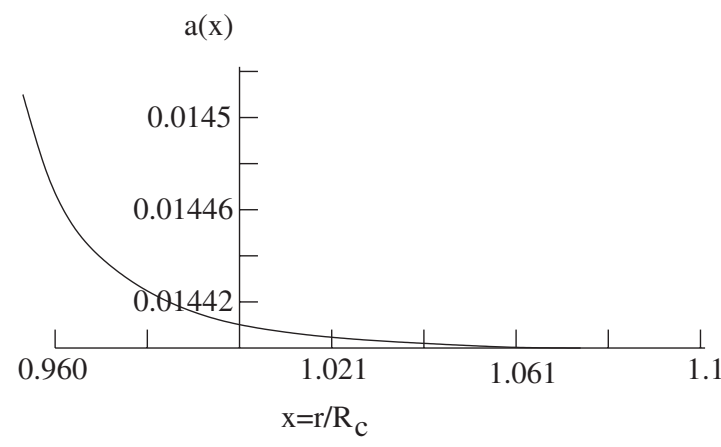

FIG. 3. The function $a(x)$ near the star surface. 


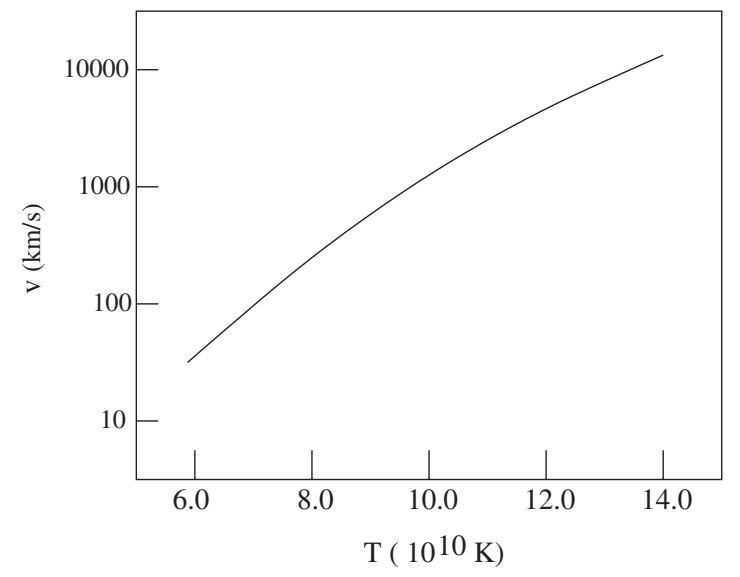

FIG. 4. Pulsar velocity vs $T$ assuming constant emission volume.

\section{CONCLUSIONS}

The basis of our present work is that for a magnetic field strength strong enough for a sizable faction of electrons produced in the modified Urca process to be in the lowest Landau level, all of the neutrinos emitted will be correlated with the direction of the magnetic field, giving a pulsar kick. The resulting velocity depends strongly on the temperature, and only indirectly on the magnetic field. Using expected values of $B\left(10^{15}\right.$ to $10^{16}$ Gauss) and $T$ (about $10^{11} \mathrm{~K}$ ), we estimate that about $40 \%$ of the electrons are in the $n=0$ Landau level, and obtain pulsar velocities of $1000 \mathrm{~km} / \mathrm{s}$ or larger.

The calculation of the asymmetric neutrino emissivity, and the resulting pulsar velocity arising from electrons being in Landau orbits in the strong magnetic field near the protoneutron star, is straightforward with the modified Urca process. Using the standard properties of protoneutron stars in the 10-20 s time interval, we find that with the electrons in Landau levels, the modified Urca process can account for the measured pulsar kicks for $T \geq 10^{11} \mathrm{~K}$, with the neutrinosphere surface just inside the protoneutron star surface. Studies [17] give $T \simeq 10^{11} \mathrm{~K}$, or greater near the protoneutron star surface. We predict a strong correlation between the protoneutron star $T$ and pulsar velocity, as well as a strong correlation between the direction of the pulsar's velocity and the direction of $B$. Since the luminosity of the pulsar is related to properties of the protoneutron star, such as strengths of $B$ and $T$, one can also expect our result to predict a correlation between $v$ and $L$ of the pulsar for high $L$.

In Fig. 5 the correlation between large $L$ and large $v$ that has been observed is shown. It is a subject for future study for us to see if our model is consistent with that observation.

Our unique prediction is that the main neutrino emission during this period when the neutrinosphere is just inside the neutron star is almost entirely asymmetric, when the

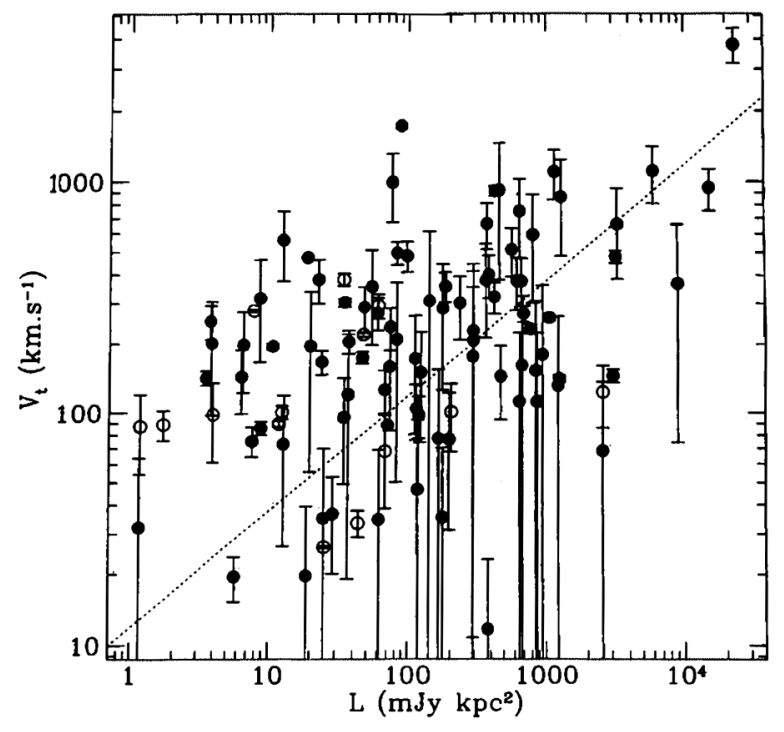

FIG. 5. Pulsar velocity vs luminosity, Ref. [4].

pulsar kicks should occur, with a strong correlation with $E_{\nu} ; \quad$ e.g., $\quad E_{\nu} \simeq 30 \mathrm{MeV} \leftrightarrow \mathrm{kT}=9.5 \mathrm{MeV}$ and $v \simeq$ $2000 \mathrm{~km} / \mathrm{s}$.

It is interesting to consider the neutrinos observed in the Kamiokande-II [26] and IBM [27] detectors from supernova SN1987A. In the analysis of the neutrino data [28], in which the Kamiokande-II and IBM data are plotted as a function of time, there might be a gap in the data, with additional neutrinos seen after $10 \mathrm{~s}$ (with the first neutrinos seen at about $1 \mathrm{~s}$ ). See Fig. 6 for KAM II and IBM results [28].

Although our agreement with the time gap seen in Fig. 6 is not statistically significant, we predict asymmetric neutrinos start to appear at about $10 \mathrm{~s}$, correlated with the $B$ direction. This should be observable in future measurements of neutrinos from supernovae with today's improved

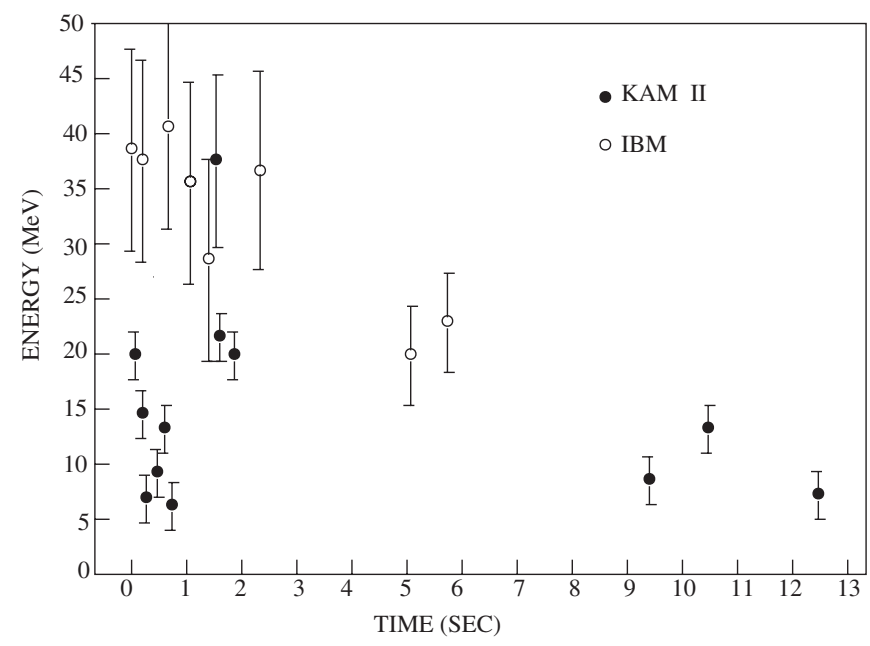

FIG. 6. Energies of neutrinos from SN1987A. 
detectors, with neutrino energies determining the pulsar velocities.

\section{ACKNOWLEDGMENTS}

This work was supported in part by DOE Contracts No. W-7405-ENG-36 and No. DE-FG02-97ER41014. The authors thank Sanjay Reddy and Richard Schirato for helpful discussions. E.M.H. and L.S.K. thank LANL for hospitality while part of this work was carried out.

\section{APPENDIX}

\section{Axial-Axial $(A-A)$ matrix elements}

The axial OPE matrix element has a direct and an exchange part,

$$
M_{A}=M_{A}^{D}+M_{A}^{E},
$$

corresponding the diagrams (1), (2), and (3) for the direct and (4), (5), and (6) for the exchange in Fig. 1. Using the notation $\chi_{n}$ for the spinor of the nucleon $n(n=1,2$ for the initial neutrons, $n=3$ for the final neutron, and $n=4$ for the produced proton), from Eqs. (2) and (6)

$$
\begin{aligned}
M_{A}^{D}= & 2 A\left[\vec{l} \cdot \vec{k} \chi_{3}^{\dagger} \sigma \cdot \vec{k} \chi_{1} \chi_{4}^{\dagger} \chi_{2}\right. \\
& \left.-\chi_{3}^{\dagger} \sigma \cdot \vec{k} \sigma \cdot l \chi_{1} \chi_{4}^{\dagger} \sigma \cdot \vec{k} \chi_{2}\right] \\
M_{A}^{E}= & -R(k) M_{A}^{D}\left(k \rightarrow k^{e}, 1 \leftrightarrow 2\right) .
\end{aligned}
$$

Using $\chi_{i} \chi_{i}^{\dagger} \equiv \Lambda_{i}$, the $D$ - $D$ product matrix element is

$$
\begin{aligned}
M_{A-A}^{D-D}= & 4 A^{2} \operatorname{Tr}\left[l \cdot k l^{\dagger} \cdot k(A 1)(A 2)+(A 3)(A 4)\right. \\
& \left.-l^{\dagger} \cdot k(A 5)(A 6)-l \cdot k(A 7)(A 8)\right],
\end{aligned}
$$

where, assuming that $\bar{k}=k-q \simeq k, \Lambda_{n}=I / 2$ and with the notation $\vec{k} \rightarrow k$,

$$
\begin{aligned}
& (A 1)=\operatorname{Tr}\left[\sigma \cdot k \Lambda_{3} \sigma \cdot k \Lambda_{1}\right]=\frac{1}{2} k^{2} \\
& (A 2)=\operatorname{Tr}\left[\Lambda_{2} \Lambda_{4}\right]=\frac{1}{2} \\
& (A 3)=\operatorname{Tr}\left[\sigma \cdot l^{\dagger} \sigma \cdot k \Lambda_{3} \sigma \cdot k \sigma \cdot l \Lambda_{1}\right]=\frac{1}{2} l_{i}^{\dagger} l_{j} k^{2} \delta_{i j} \\
& (A 4) \simeq(1 A)=\frac{1}{2} k^{2} \quad(A 6)=\operatorname{Tr}\left[\sigma \cdot k \Lambda_{2} \Lambda_{4}\right]=0 \\
& (A 8)=\operatorname{Tr}\left[\sigma \cdot k \Lambda_{4} \Lambda_{2}\right]=0 .
\end{aligned}
$$

From this we find

$$
\begin{aligned}
& M_{A-A}^{D-D}=A^{2} \operatorname{Tr}\left[l_{i}^{\dagger} l_{j}\right] k^{2}\left(k_{i} k_{j}+k^{2} \delta_{i j}\right) \\
& M_{A-A}^{E-E}=A^{2} R(k)^{2} \operatorname{Tr}\left[l_{i}^{\dagger} l_{j}\right]\left(k^{e}\right)^{2}\left(k_{i}^{e} k_{j}^{e}+\left(k^{e}\right)^{2} \delta_{i j}\right) .
\end{aligned}
$$

Using $\bar{k} \simeq k, \bar{k}^{e} \simeq k^{e}$, the two direct-exchange product matrix elements can be written as (note $M_{A-A}^{E-D}=$ $\left.M_{A-A}^{D-E}\left(k \leftrightarrow k^{e}\right)\right)$

$$
\begin{aligned}
M_{A-A}^{D-E}= & -4 A^{2} R(k) \sum_{\text {spins }}\left[l^{\dagger} \cdot k l \cdot k^{e} D E 1+D E 2\right. \\
& \left.-l^{\dagger} \cdot k D E 3-l \cdot k^{e} D E 4\right],
\end{aligned}
$$

with (assuming $\Lambda_{n}=I / 2$ for all nucleons)

$$
\begin{aligned}
D E 1 & =\frac{1}{16} \operatorname{Tr}\left[\sigma \cdot k \sigma \cdot k^{e}\right]=\frac{1}{8} k \cdot k^{e} \\
D E 2 & =\frac{1}{16} \operatorname{Tr}\left[\sigma \cdot l^{\dagger} \sigma \cdot k \sigma \cdot k^{e} \sigma \cdot l \sigma \cdot k \sigma \cdot k^{e}\right] \\
& =\frac{1}{8} l_{i}^{\dagger} l_{j}\left[-k \cdot k^{e}\left(k_{i} i_{j}^{e}+k_{i}^{e} i_{j}\right)+k_{i} k_{j}\left(k^{e}\right)^{2}+k_{i}^{e} k_{j}^{e}(k)^{2}-\left(k \times k^{e}\right)_{i}\left(k \times k^{e}\right)_{j}+\delta_{i j}\left(k \times k^{e}\right)^{2}\right] \\
D E 3 & =\frac{1}{16} \operatorname{Tr}\left[\sigma \cdot k \sigma \cdot k^{e} \sigma \cdot l \sigma \cdot k^{e}\right]=\frac{1}{8}\left(2 k \cdot k^{e} l \cdot k^{e}-l \cdot k\left(k^{e}\right)^{2}\right) \\
D E 4 & =\frac{1}{16} \operatorname{Tr}\left[\sigma \cdot l^{\dagger} \sigma \cdot k \sigma \cdot k^{e} \sigma \cdot k\right]=\frac{1}{8}\left(2 k \cdot k^{e} l^{\dagger}-l^{\dagger} \cdot k^{e}(k)^{2}\right) .
\end{aligned}
$$

To obtain Eq. (A8) we use the trace relationships

$$
\begin{aligned}
\operatorname{Tr}[\sigma \cdot A \sigma \cdot B \sigma \cdot C \sigma \cdot D]= & 2(A \cdot B C \cdot D \\
& -A \times B \cdot C \times D),
\end{aligned}
$$

$\operatorname{Tr}[\sigma \cdot A \sigma \cdot B \sigma \cdot C \sigma \cdot D \sigma \cdot E \sigma \cdot F]$

$$
\begin{aligned}
= & 2 E \cdot F(A \cdot B C \cdot D-A \times B \cdot C \times D) \\
& -2[A \cdot B E \times F \cdot C \times D+C \cdot D E \times F \cdot A \times B \\
& -((A \times B) \times(C \times D)) \cdot E \times F] .
\end{aligned}
$$

From this, defining $M_{A A}^{D E}=M_{A-A}^{D-E}+M_{A-A}^{D-E}$ we obtain

$$
\begin{aligned}
M_{A A}^{D E}= & -A^{2} R(k) \operatorname{Tr}\left[l_{i}^{\dagger} l_{j}\right]\left[-\frac{5}{2} k \cdot k^{e}\left(k_{i} k_{j}^{e}+k_{i}^{e} k_{j}\right)\right. \\
& +2 k_{i} k_{j}\left(k^{e}\right)^{2}+2 k_{i}^{e} k_{j}^{e}(k)^{2}+\left(k \cdot k^{e}\right)^{2} \delta_{i j} \\
& \left.-\left(k \times k^{e}\right)_{i}\left(k \times k^{e}\right)_{j}\right] .
\end{aligned}
$$

\section{Lepton traces}

The lepton trace, $\operatorname{Tr}\left[l_{i}^{\dagger} l_{j}\right]$, from Eq. (2), with the neutrino in a standard Dirac state, $u^{s}\left(q^{\nu}\right)$, and the electron in the lowest Landau level, $\left.\Psi\left(q^{e}\right)=i(\sqrt{\gamma})^{-1}\right) \times$ $e^{-\left(q_{\perp}^{e}\right)^{2} /(2 \gamma)} u^{-}\left(q^{e}\right)$, with the electron momentum along the direction of the magnetic field, its spin in the opposite 
direction, is defined as

$$
\operatorname{Tr}\left[l_{i}^{\dagger} l_{j}\right]=\frac{2}{\gamma} e^{-\left(q_{\perp}^{e}\right)^{2} / \gamma} \operatorname{Tr}\left[\gamma_{i} \phi^{\nu} \gamma_{j}\left(I-\gamma_{5}\right) u^{-}\left(q^{e}\right) \bar{u}^{-}\left(q^{e}\right)\right] .
$$

Using $m_{e} \ll E_{e}$, and dropping terms in $\left(q^{0}\right)^{\nu}$, which do not give rise to momentum asymmetry,

$$
\begin{aligned}
\left.\gamma_{i} \phi^{\nu} \gamma_{j}\right|_{i \neq j} & =-\left(q^{i}\right)^{\nu} \gamma_{j}-\left(q^{j}\right)^{\nu} \gamma_{i} \\
\gamma_{i} \phi^{\nu} \gamma_{i} & =\not q^{\nu}-2\left(q^{i}\right)^{\nu} \gamma_{i} \\
\left(I-\gamma_{5}\right) u^{-}\left(q^{e}\right) \bar{u}^{-}\left(q^{e}\right)= & \frac{E^{e}}{2}\left[\gamma^{o}+\gamma^{o} \gamma^{5}+\gamma^{5} \gamma^{3}-\gamma^{3}\right] \\
& \times\left(\hat{q}^{e}=\hat{B}=\hat{z}\right) \\
\int d^{2} q_{\perp} \frac{1}{\gamma} e^{-\left(q_{\perp}\right)^{2} / \gamma} & =2 \pi .
\end{aligned}
$$

An essential part of our work is that $(I-$ $\left.\gamma_{5}\right) u^{-}\left(q^{e}\right) \bar{u}^{-}\left(q^{e}\right)$ vanishes if the electron momentum is opposite to the $B$-field, and that only electrons in the lowest Landau level in the direction of the $B$-field contribute to the emissivity. From Eqs. (A12) and (A13) we find for the electron in the lowest Landau level

$$
\begin{aligned}
\int d^{2} q_{\perp}^{e} \operatorname{Tr}\left[l_{i}^{\dagger} l_{j}\right] \simeq & 8 \pi E^{e}\left[\left(q^{\nu}\right)^{j} \delta_{i 3}+\left(q^{\nu}\right)^{i} \delta_{j 3}-\delta_{i j}\left(q^{\nu}\right)^{3}\right] \\
& \times\left(\hat{q}^{e}=\hat{B}=\hat{z}\right) .
\end{aligned}
$$

\section{Angular integration}

Using $k=p_{1}-p_{3}, k^{e}=p_{2}-p_{1}$ as independent variables (see text for discussion), and defining $\mathbb{l} \equiv$ $\int d \Omega_{k} \int \frac{d \Omega_{k^{e}}}{4 \pi}$. We need the following angular integrals:

$$
\begin{gathered}
\iint k_{i}=\iint k_{i}^{e}=0 \quad \iint k \cdot A k \cdot B=\frac{4 \pi k^{2}}{3} A \cdot B \\
\iint k \cdot A k \cdot B k \cdot C k \cdot D=\frac{4 \pi k^{4}}{15}(A \cdot B C \cdot D+A \cdot C B \cdot D+A \cdot D B \cdot C) \\
\iint k^{2}=4 \pi k^{2} \quad \iint\left(k_{z}\right)^{2}=\frac{4 \pi}{3} k^{2} \quad \iint\left(k \cdot k^{e}\right)^{2}=\frac{4 \pi}{3} k^{2}\left(k^{e}\right)^{2} \quad \iint k \cdot k^{e} k_{z} k_{z}^{e}=\frac{4 \pi}{9} k^{2}\left(k^{e}\right)^{2} \\
\iint\left(\left(k \times k^{e}\right)_{z}\right)^{2}=\frac{8 \pi}{9} k^{2}\left(k^{e}\right)^{2} \quad \iint k \cdot k^{e} k_{z} k^{e} \cdot q=\frac{4 \pi}{9} k^{2}\left(k^{e}\right)^{2} q_{z} \quad \iint k_{z}^{e} k^{e} \cdot q=\frac{4 \pi}{3}\left(k^{e}\right)^{2} q_{z} \\
\iint k \times k^{e} \cdot q\left(k \times k^{e}\right)_{z}=\frac{8 \pi}{9} k^{2}\left(k^{e}\right)^{2} q_{z} \quad \iint\left(k \times k^{e}\right)_{i} k \cdot k^{e}=0 .
\end{gathered}
$$

[1] J. N. Bahcall and R.A. Wolf, Phys. Rev. 140, B1452 (1965).

[2] E. G. Flowers, P. G. Sutherland, and J. R. Bond, Phys. Rev. D 12, 315 (1975).

[3] B. L. Friman and O. V. Maxwell, Astrophys. J. 232, 541 (1979).

[4] B. M. S. Hansen and E. S. Phinney, Mon. Not. R. Astron. Soc. 291, 569 (1997).

[5] J. Murphy, A. Burrows, and A. Heger, Astrophys. J. 615, 460 (2004).

[6] H-Th. Janka, L. Sheck, K. Kifonidis, E. Müller, and T. Plewa, arXiv:astro-ph/0408439.

[7] Chen Wang, Dong Lai, and J. L. Han, Astrophys. J. 656, 399 (2007).

[8] H-Th. Janka, A. Marek, and F-S. Kitaura, arXiv:0706.3056.

[9] O. F. Dorofeev et al., Sov. Astron. Lett. 11, 123 (1985).

[10] C. J. Horowitz and J. Piekarwitz, Nucl. Phys. A640, 281 (1998).
[11] C. J. Horowitz and G. Li, Phys. Rev. Lett. 80, 3694 (1998).

[12] D. Lai and Y-Z Qian, arXiv:astro-ph/9802345.

[13] P. Arras and D. Lai, Phys. Rev. D 60, 043001 (1999).

[14] A. Kusenko and G. Segre, Phys. Lett. B 396, 197 (1997).

[15] G. M. Fuller, A. Kusenko, I. Mocioiu, and S. Pascoli, Phys. Rev. D 68, 103002 (2003).

[16] M. Barkovich, J.C. D'Olivo, R. Montemayor, and J.F. Zanella, Phys. Rev. D 66, 123005 (2002).

[17] M. Barkovich, J.C. D'Olivo, and R. Montemayor, arXiv:hep-ph/0503113.

[18] A. A. Aguilar-Arevalo et al. (MiniBooNE Collaboration), Phys. Rev. Lett. 98, 231801 (2007).

[19] M. H. Johnson and B. A. Lippman, Phys. Rev. 76, 828 (1949).

[20] J. J. Matese and R.F. O'Connel, Phys. Rev. 180, 1289 (1969).

[21] L. S. Kisslinger, Mod. Phys. Lett. A 22, 2071 (2007).

[22] A study by E. M. Henley, M. B. Johnson, and L.S. Kisslinger (unpublished). 
[23] P. J. Schinder and S. L. Shapiro, Astrophys. J. 259, 311 (1982).

[24] H-T. Janka and G. G. Raffelt, Phys. Rev. D 59, 023005 (1998).

[25] M. Prakash, J. M. Lattimer, J. A. Pons, A. W. Steiner, and
S. Reddy, Lect. Notes Phys. 578, 364 (2001).

[26] K. Hirata et al., Phys. Rev. Lett. 58, 1490 (1987).

[27] R. M. Bionta et al., Phys. Rev. Lett. 58, 1494 (1987).

[28] K. Hirata et al., Phys. Rev. D 38, 448 (1988). 